\title{
Treatment of Subacute Sclerosing Panencephalitis with Human Leukocyte Interferon
}

\author{
Naoki Mizutani, Mitsunobu Miyazu, Mitsuo Maehara, \\ Shin Isomura, Kazuyoshi Watanabe and Jiro \\ IMANISHI* \\ Department of Pediatrics, Nagoya University School of \\ Medicine, Nagoya 466, and *Department of Microbiology, \\ Kyoto Prefectual University of Medicine, Kyoto 602
}

\begin{abstract}
Mizutani, N., Miyazu, M., Maehara, M., Isomura, S., Watanabe, K. and Imanishi, J. Treatment of Subacute Sclerosing Panencephalitis with Human Leukocyte Interferon. Tohoku J. exp. Med., 1985, 146 (3), 277-284—In five patients with subacute sclerosing panencephalitis (SSPE), human leukocyte interferon (IFN) therapy was tried. IFN was administered intramuscularly, intravenously, and intrathecally. The total dose of administered IFN ranged from $13 \times 10^{6}$ to $116.92 \times 10^{6} \mathrm{IU}$. There were no severe side effects except for temporary high fever and vomiting. Observation of the clinical course were made for 26-60 months. At the beginning of the treatment, one of the patients was at the first stage of Jabbour's classification and the other four at the second stage. No clinical improvement was observed and the clinical course was progressive in all patients. At present, one of the patients was at the second stage and the other four were at the fourth stage. EEGs showed progressive deterioration, and cranial CT scan demonstrated progressive cortical atrophy and ventricular enlargement. Measles antibody titers in the serum and CSF also unchanged. On the other hand, permeability of IFN at blood-brain barrier (BBB) was relatively good.

blood-brain barrier; interferon; subacute sclerosing panencephalitis (SSPE)
\end{abstract}

Subacute sclerosing panencephalitis (SSPE) is an uncommon, progressive central nervous system disease in children and young adolescents. It is considered to be slow virus infection caused by measles-like paramyxovirus (Connolly et al. 1967 ; Baublis and Payne 1968; Horta-Barbosa et al. 1969). However, the pathogenesis of SSPE is still unclear (Burnet 1968; Saunders et al. 1969 ; Brody and Detels 1970 ; Allen et al. 1973; Ahmed et al. 1974; Oldstone et al. 1975). Up to the present, there have been some attempts to treat the patients with SSPE with the progress of the research on its pathogenesis (Haslam et al. 1969; Kertesz et al. 1970 ; Robertson et al. 1979). But the effectiveness of these agents is still unsettled. Recently, there are some descriptions which report the effect of transfer factor (TF) (Vandvik et al. 1973) and inosiplex therapy on the patient

Received September 8, 1984 ; accepted for publication January 2, 1985. 
with SSPE (Streletz and Cracco 1977 ; Huttenlocher and Mattson 1979 ; DuRant et al. 1982 ; Dyken et al. 1982 ; Jones et al. 1982). However, their effects are also unclear (Silverberg et al. 1979; Haddad and Risk 1980).

Since 1978, we have been treating the patient with SSPE with interferon (IFN). The aim of this study is to report the results of IFN therapy in five patients with SSPE, and to investigate the permeability of IFN at blood-brain barrier (BBB) after intramuscular or intravenous IFN administration.

\section{Cases and Methods}

Five patients with SSPE were studied. Their clinical features and labolatory findings are summarized in Table 1. The diagnosis of SSPE was based on their characteristic clinical features, periodic myoclonus, periodic bursts in electroencephalogram (EEG), elevated measles antibody titers in the cerebrospinal fluid (CSF) and serum, and increased CSF IgG content.

All patients were male aged 6 to 12 years. One of the patient (case 1) was at the end of the first stage by Jabbour's classification at the beginning of the IFN treatment, and the other four were at the second stage. The clinical course was very slowly progressive in case 1 , and relatively progressive in the other four.

The patients presented with generalized tonic and clonic convulsion, gait disturbance, changes of the character or mental deterioration. They caught measles at the age of 6 to 12 months. The age at the onset of SSPE ranged from 5 to 12 years, and the interval between measles and the onset of SSPE ranged from 4 to 12 years. The EEGs revealed typical periodic bursts in all cases. The cranial CT scan performed at the initial diagnosis revealed low density areas in the basal ganglia in case 3 , but were normal in other four.

Human leukocyte IFN was obtained from Professor T. Kishida (Department of Microbiology, Kyoto Prefectual University of Medicine). Human leukocyte IFN was produced by in vitro induction in human buffy caot leukocytes by the method of Cantell and Hirvonen (1977). As a rule, IFN was administred intamuscularly at a dose of 1.0$1.5 \times 10^{6}$ unit per day, at a dose of $0.3-1.5 \times 10^{6}$ unit per day intravenously, and $10-120 \times$ $10^{3}$ unit per day intrathecally.

The assessment of the clinical effect was based on the changes of clinical symptoms, EEG findings, cranial CT scan findings, and measles antibody titers in the CSF and serum before and after IFN administration.

Furthermore, to investigate the permeability of IFN at BBB after intramuscular or intravenous administration, serial changes of IFN titers were studied in CSF and serum simultaneously. IFN assays in CSF and serum were performed according to the cytopathic effect inhibition method using FL cells and vesicular stomatitis virus (VSV) as a challenge virus (Havell and Vilcek 1972).

\section{RESUlts}

Therapeutic effect of IFN on patients with SSPE (Table 2)

From October 2, 1978 through June 29, 1982, a total of 13.0-116.92 $\times 10^{6}$ unit of IFN was given to each patient. Period of IFN therapy ranged from 3-26 months. The observation of the clinical course was made for 26-60 months. High fever was observed $3 \mathrm{hr}$ after intramuscular or intravenous IFN administration in case 2 , but was temporary. There were no other severe side effects. Intrathecal administration of IFN was made in three cases. 
Treatment of SSPE with Interferon

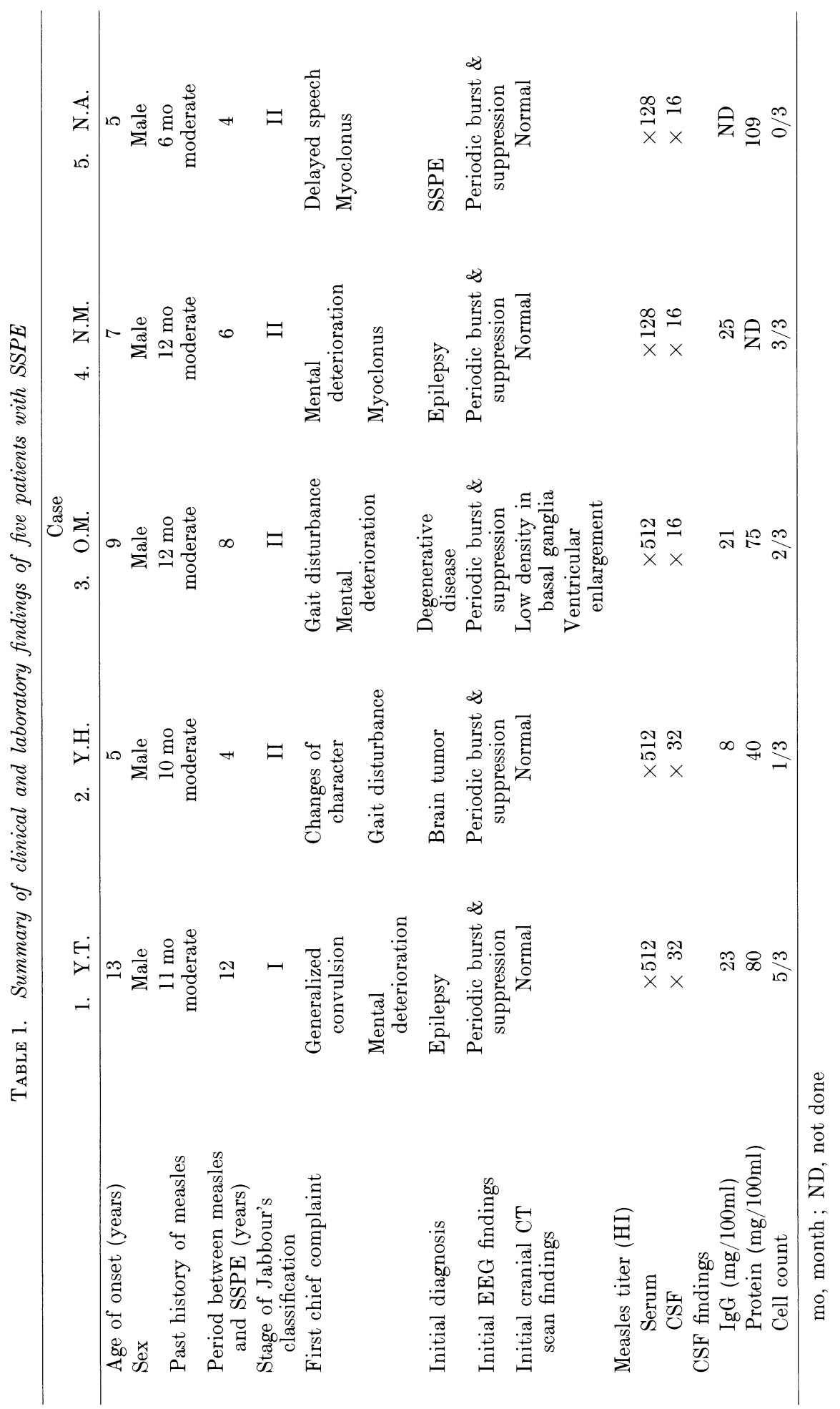


N. Mizutani et al.

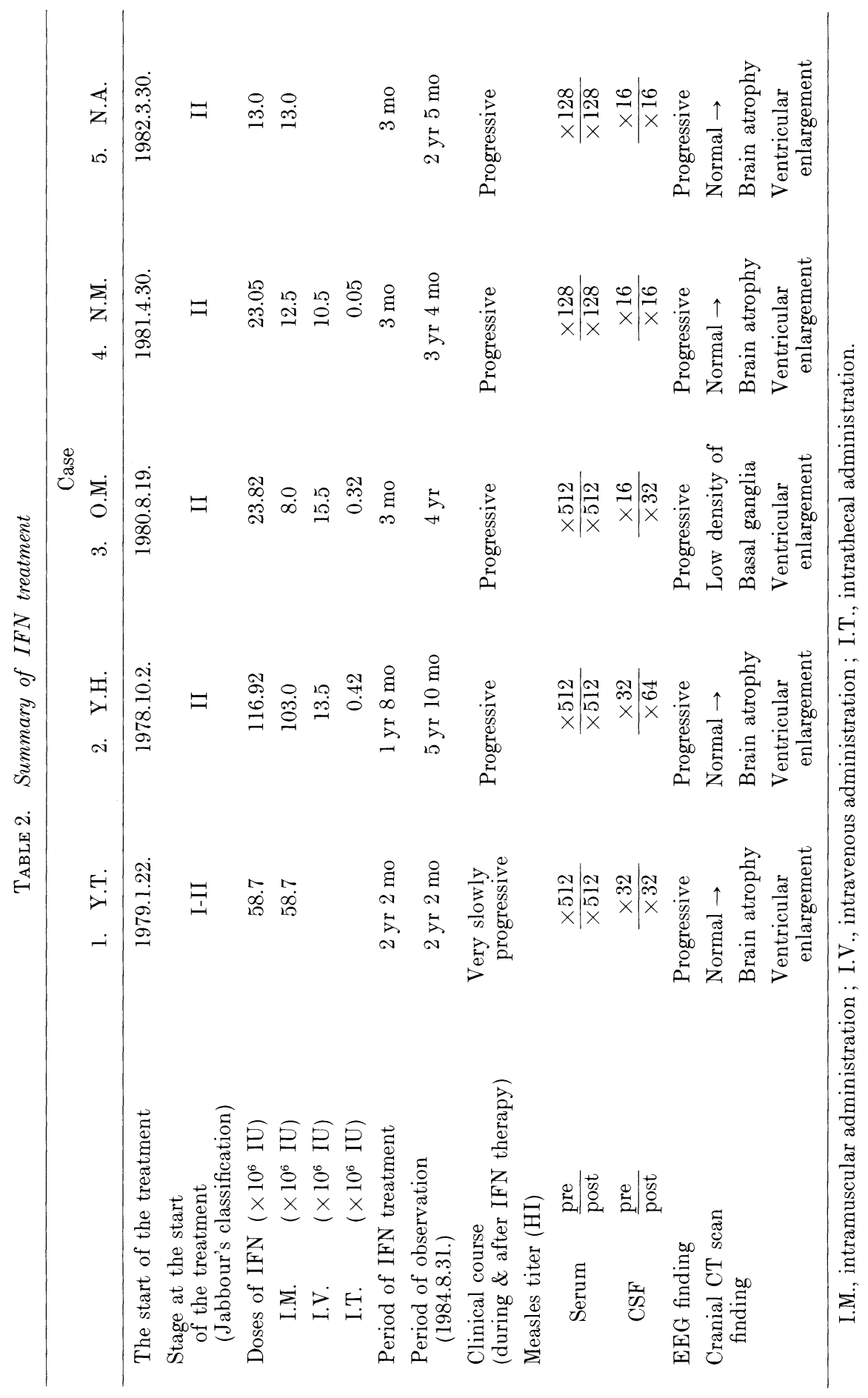


Clinical course: No clincal improvement was observed in all cases. Case 1 showed very slowly progressive course ; simple speech and writing, and supported gait were possible. The other four cases deteriorated moderately. By the time of this writing, four cases except for case 1 had progressed to the fourth stage by Jabbour's classification. They were all in vegetative state without spontaneous speech and response to stimulation. The interval between myoclonic jerks was prolonged and myoclonus was diminished in two cases.

Laboratory findings: In all cases, there was no improvement in EEG findings. The EEG performed at the diagnosis showed periodic bursts, typical for SSPE in all cases. 26-60 month observation of EEG findings during and after IFN treatment revealed a progressive disorganization and slowing of the basic rhythm. Furthermore, sleep stage differentiation became unclear in all cases.

Cranial CT scans performed before IFN therapy were almost normal except for case 3, which showed slight enlargement of the lateral ventricles and slightly low density areas of the basal ganglia. In spite of IFN therapy, cranial CT scan performed 24-48 months after the start of IFN therapy revealed marked dilatation of the lateral and the third ventricles, severe enlargement of Sylvian fissures, and severe cortical atrophy.

Measles antibody titers in CSF and serum did not show any changes after IFN therapy. There was neither granulocytepenia nor thrombocytopenia. Liver function tests and immunological examination were also within normal limits.

IFN titers and permeability of IFN at BBB (Fig. 1)

Intramuscular and intravenous administration of IFN resulted in a gradual elevation of its serum levels with the peak level one and a half hour after the

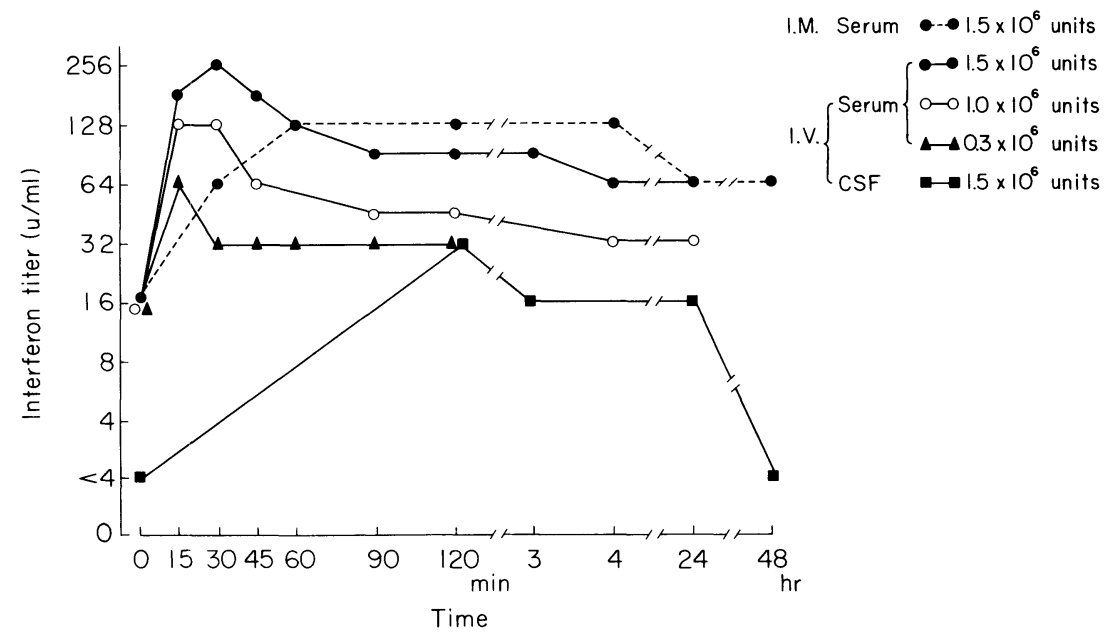

Fig. 1. Interferon titers of serum and CSF after intramuscular (I.M.) or intravenous (I.V.) administration of human leukocyte interferon. 


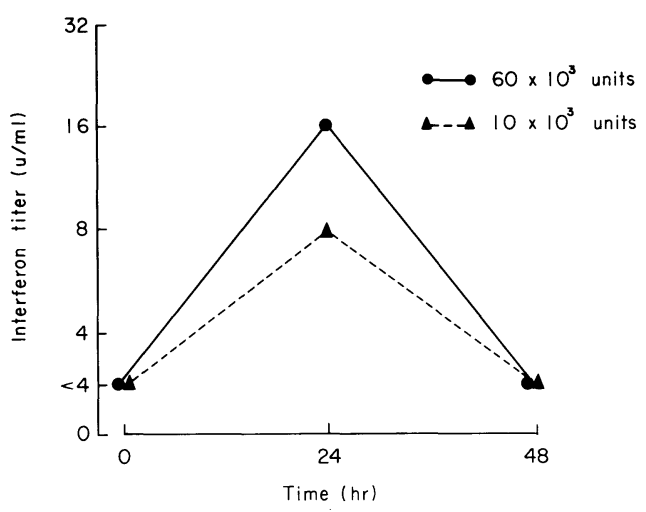

Fig. 2. Interferon titers of CSF after intrathecal administration of human leukocyte interferon.

administration respectively. IFN titers in CSF was not detected before IFN administration, but raised to a quarter to an eighth of that of serum $24 \mathrm{hr}$ later, but was diminished rapidly. This indicates that the permeability of intramuscularly or intravenously administered IFN at BBB is relatively good. On the other hand, serum IFN titers diminished gradually.

Intrathecal IFN administration was performed in three cases. It was associated with temporary high fever and vomiting in all cases. Because of these side effects, a large amount of IFN could not be given at a time.

\section{Discussion}

Subacute sclerosing panencephalitis (SSPE) is a rare progressive disorder of the central nervous system associated with persistent slow measles-like virus infection (Connolly et al.1967; Baublis and Payne 1968; Horta-Barbosa et al. 1969). There are some hypotheses about the route of the infection, but the details of the pathogenesis are still unclear (Burnet 1968; Saunders et al. 1969; Brody and Detels 1970 ; Allen et al. 1973; Ahmed et al. 1974; Oldstone et al. 1975).

Recently, there are some reports that isoprinosine (inosiplex)(Streletz and Cracco 1977; Huttenlocher and Mattson 1979; Dyken et al. 1982 ; Jones et al. 1982 ; DuRant et al. 1982) or TF (Vandvik et al. 1973) is effective. On the other hand, there are a few reports to the contrary (Silverberg et al. 1979; Haddad and Risk 1980).

The persistence of virus-infected cells and the presence of high levels of serum and CSF antibody to measles antigen have led to a speculation that immunological mechanism may involved. Therefore, we expected the effect of IFN, which not only has antiviral properties but also modifies the immune response and activates natural killer cells. Up to the present, there have been only a few papers concerning the effects of IFN on SSPE (Behan 1981; Bartram et al. 1982). But the decision on the effects of IFN treatment must be made carefully, in view 
of reported occurences of spontaneous long-term improvement or remission (Risk and Haddad 1979).

In this study, although there seemed to be some improvement of general condition and cessation of the progress of disease stage, no difinite improvement was observed in clinical symptoms or laboratory examinations. If IFN therapy had been started in the earlier stage, some effects might have been observed. But once clinical symptoms appear in the central nervous system disorders, the brain damage is usually relatively advanced and irreversible.

In the present study, there demonstrated relatively good permeability of IFN at BBB. De Clercq et al. (1975) reported that intrathecal administration of leukocyte IFN was ineffective for neonatal herpes encephalitis, and the permeability of IFN at BBB was bad. Billiau (1981) demonstrated that very little, if any, IFN penetrates into the brain when given intrathecally. On the other hand, this study showed that disappearance of IFN activity from CSF was rapid. This phenomenon may be attributable to a rapid turn-over of CSF in children.

\section{References}

1) Ahmed, A., Strong, D.M., Sell, K.W., Thurman, G.B., Knudsen, R.C., Wister, R., Jr. \& Grace, W.R. (1974) Demonstration of a blocking factor in the plasma and spinal fluid of patients with subacute sclerosing panencephalitis: I. Partial characterization. $J$. $\operatorname{exp.~Med.,~139,~902-924.~}$

2) Allen, J., Oppenheim, J., Brody, J.A. \& Miller, J. (1973) Labile inhibitor of lymphocyte transformation in plasma from a patient with subacute sclerosing panencephalitis. Infec. Immunity, 8, 80-82.

3) Bartram, C.R., Henke, J., Treuner, J., Basler, M., Esch, A. \& Mortier, W. (1982) Subacute sclerosing panencephalitis in a brother and sister: Therapeutic trial of fibroblast interferon. Europ. J. Pediat., 138, 187-190.

4) Baublis, J.V. \& Payne, F.E. (1968) Measles antigen and syncytium formation in brain cell cultures from subacute sclerosing panencephalitis (SSPE). Proc. Soc. exp. Biol. Med., 129, 593-597.

5) Behan, P.O. (1981) Interferon treatment of subacute sclerosing panencephalitis. Lancet, I, 1059-1060.

6) Billiau, A. (1981) Interferon therapy: Pharmakokinetic and pharmacological aspects. Arch. Virol., 67 121-133.

7) Brody, J.A. \& Detels, R. (1970) Subacute sclerosing panencephalitis: A zoonosis following aberrant measles. Lancet, II, 500-501.

8) Burnet, F.M. (1968) Measles as an index of immunological function. Lancet, II, 610-613.

9) Cantell, K. \& Hirvonen, S. (1977) Preparation of human leukocyte interferon for clinical use. Texas Rep. Biol. Med., 35, 138-144.

10) Connolly, J.H., Allen, I.V., Hurwitz, L.J. \& Miller, J.H.D. (1967) Measles-virus antibody and antigen in subacute sclerosing panencephalitis. Lancet, I, 542-544.

11) De Clercq, E., Edy, V.D., DeVlinger, H., Eeckels, R. \& Desmyter, J. (1975) Intrathecal administration of interferon in neonatal herpes. J. Pediatr., 86, 736-739.

12) DuRant, R.H., Dyken, P.R. \& Swift, A.V. (1982) The influence of inosiplex treatment on the neurological disability of patients with subacute sclerosing panencephalitis. J. Pediat., 101, 288-293.

13) Dyken, P.R., Swift, A.V. \& DuRant, R.H. (1982) Long-term follow-up of patients 
with subacute sclerosing panencephalitis treated with inosiplex. Ann. Neurol., 11, 359-364.

14) Haddad, F.S. \& Risk, W.S. (1980) Isoprinosine treatment in 18 patients with subacute sclerosing panencephalitis: A controlled study. Ann. Neurol., 7, 185-188.

15) Haslam, M.H.A., McQuillen, M.P. \& Clark, D.B. (1969) Amantadine therapy in subacute sclerosing panencephalitis: A preliminary report. Neurology, 19, 10801086.

16) Havell, E.A. \& Vilcek, J. (1972) Production of high-titered interferon in cultures of human diploid cells. Antimicrob. Agents Chemother., 2, 476-484.

17) Horta-Barbosa, L., Fuccillo, D.A., Sever, J.L. \& Zeman, W. (1969) Subacute sclerosing panencephalitis : Isolation of measles virus from brain biopsy. Nature, 221, 974.

18) Huttenlocher, P.R. \& Mattson, R.H. (1979) Isoprinosine in subacute sclerosing panencephalitis. Neurology, 29, 763-771.

19) Jones, C.E., Dyken, P.R., Huttenlocher, P.R., Jabbour, J.T. \& Maxwell, K.W. (1982) Inosiplex therapy in subacute sclerosing panencephalitis: A multicentre, nonrandomized study in 98 patients. Lancet, I, 1034-1036.

20) Kertesz, A., Veidlinger, O.P. \& Furesz, J. (1970) Subacute sclerosing panencephalitis: Studies of two cases treated with 5-bromo-2-deoxyuridine. Canad. med. Ass. J., 102, 1264-1269.

21) Oldstone, M.B.A., Bokisch, V.A., Dixson, F.J., Horta-Barbosa, L., Fuccillo, D \& Sever, J.L. (1975) Subacute sclerosing panencephalitis: Destruction of human brain cells by antibody and complement in an autologous system. Clin. Immunol. Immunopathol., 4, 52-58.

22) Risk, W.S. \& Haddad, F.S. (1979) The variable natural history of subacute sclerosing panencephalitis-A study of 118 cases from the middle east. Arch. Neurol., 36, 610614.

23) Robertson, W.C., Jr., Clark, D.B. \& Markesbery, W.R. (1979) Review of 38 cases of subacute sclerosing panencephalitis : Effect of amantadine on the natural course of the disease. Ann. Neurol., 8, 422-425.

24) Saunders, M., Knowles, M., Chambers, M.E., Caspary, E.A., Gardner-Medwin, D \& Walker, P. (1969) Cellular and humoral responses to measles in subacute sclerosing panencephalitis. Lancet, I, 72-74.

25) Silverberg, R., Brenner, T \& Swift, A.V. (1979) Inosiplex in the treatment of subacute sclerosing panencephalitis. Arch. Neurol., 36, 374-375.

26) Streletz, L.J. \& Cracco, J. (1977) The effect of isoprinosine in subacute sclerosing panencephalitis (SSPE). Ann. Neurol., 1, 183-184.

27) Vandvik, B., Froland, S.S., Hoyeraal, H.M., Stien, R. \& Degre, M. (1973) Immunological features in a case of subacute sclerosing panencephalitis: Treated with transfer factor. Scand. J. Immunol., 2, 367-374. 